\title{
Emotional responses in monkeys inhibited with electrical stimulation
}

ROD PLOTNIK and JOSE M.R. DELGADO, Yale University School of Medicine, New Haven, Conn. 06510

Electrodes were implanted in the caudate nucleus, putamen, and other areas of adult rhesus monkeys. Electrical stimulation of some of these areas inhibited the specific emotional responses of threatening or grimacing without disrupting consummatory, instrumental, locomotor, or grooming behavior.

There is a question as to whether or not emotional responses can be suppressed by electrical stimulation of the brain (ESB) without impairment of other behaviors. Previous studies that have reported inhibition of emotional responses by ESB also reported longer latencies for initiation of movement (Siegel \& Flynn, 1968), suppression of eating (Fonberg \& Delgado, 1961; Rubinstein \& Delgado, 1963; Fonberg, 1963; Egger \& Flynn, 1963), or did not report effects on eating (Delgado, 1964). If ESB suppressed eating and aggression, then facial movement in general might be inhibited. The present experiment presents evidence for ESB inhibiting only specific emotional responses.

\section{METHOD}

Four mature rhesus monkeys were each implanted stereotaxically with four to six permanent electrode assemblies as previously described (Delgado, 1955). Throughout testing, monkeys were observed over closed-circuit TV, since E presence can bias results (Plotnik et al, in press). A total of 154 areas of the brain were explored to locate sites from which ESB inhibited emotional responses of threatening/dominant gesture, in which monkey opens mouth without exposing teeth and stares with occasional head-bobbing and low vocalizations at stimulus, or of grimacing/submissive gesture, in which monkey retracts upper and lower lips, exposing teeth and sometimes vocalizing in presence of threatening stimulus. To check for possible paralysis of facial and masticatory muscles during ESB, monkeys, restrained and unrestrained, were tested for ability to open mouth, chew a banana, and drink water. Each monkey, unrestrained in a cage $2 \frac{1}{2} \times 3 \frac{1}{2} \times 5 \mathrm{ft}$ long, was observed during ESB and tested on a previously learned task of lever pressing for food pellets (one pellet for five presses), to measure disruptive or competing motor responses, effects on locomotion, and ability to perform an instrumental task. The effects of ESB were also tested on a second instrumental response of lever pressing to avoid shock that occurred every $5 \mathrm{sec}$ unless restrained monkey pressed lever, which postponed shock for $15 \mathrm{sec}$. During testing of instrumental responses, ESB occurred $5 \mathrm{sec}$ on $/ 5 \mathrm{sec}$ off throughout the 5-min food and shock sessions. Finally, effects of ESB were tested on unrestrained monkey's ability to perform well-coordinated motor movements as seen when one animal grooms another. ESB occurred $(5 \sec$ on $/ 5 \sec$ off $)$ at initiation of and until grooming or self-grooming terminated.

During testing of emotional responses, the dominant monkey was restrained and its chair placed against the glass front of a cage, $2 \frac{1}{2} \times 2 \frac{1}{2} \times 3 \mathrm{ft}$ long, that contained a submissive monkey. A simultaneous $0.2-\mathrm{sec}$ shock to tail and seat of restrained monkey and feet of caged monkey was followed by $5 \mathrm{sec}$ of ESB to operated dominant monkey. Normally, within $5 \mathrm{sec}$ after shock, the dominant monkey threatened the caged submissive monkey. Inhibition occurred if there were no threats during the 5-sec ESB period. Pilot work indicated that shocking both monkeys together in a cage elicited fighting that was too severe for long-term testing and often damaged electrode assemblies. By restraining dominant monkey, both problems were solved without change in frequency or direction of threat behavior toward caged monkey.

Two of the four monkeys were submissive, that is, shock elicited grimacing, crouching, or freezing in the cage with any other monkey that severely attacked the operated monkey. To elicit grimacing reliably and eliminate possible injury to operated monkey, it was restrained in a chair and tested with a 12-in.-high troll doll that swung from $6 \mathrm{ft}$ away into the front of monkey's chair. At initiation of doll's swing, the monkey received a $0.2-\mathrm{sec}$ shock between its tail and seat of chair. Without shock, the approaching doll evoked grimacing that habituated in from 10 to 40 trials, while with paired shock grimacing did not habituate. At the end of its swing, the doll contacted the chair twice and normally the monkey grimaced and vocalized each time. ESB $(5 \mathrm{sec})$ began with first contact and inhibition was present if no grimacing occurred on either of doll's contacts with chair. A doll was used as the threatening stimulus instead of a dominant monkey because, in pilot work, it was found that if the restrained submissive monkey was placed in front of a restrained or caged dominant partner, following shock the dominant monkey threatened while the submissive monkey grimaced the first few trials but thereafter sat in the chair with its head turned to one side, avoiding eye contact with the dominant monkey.

During control testing without ESB, the percentage of shock-evoked threats or grimaces was very reliable and never below $90 \%$ per 60 trials, with $30 \mathrm{sec}$ between each trial.

All inhibitory points in the restrained monkey were tested for elicitation of after-discharge activity, which is high-amplitude, rhythmical electrical activity continuing after termination of electrical stimulation. Since spread of after-discharges can excite other brain areas, behavioral effects seen during after-discharges cannot be attributed solely to area originally stimulated. Thus, behavioral responses occurring during after-discharges were not included in the following data.

Each cerebral site was stimulated in the restrained monkey with increasing intensity steps (0.05-1.5 mA) until either evidence for inhibition was found or some motor response occurred. If evidence for inhibition was found, 60 trials were given and then monkey was tested on consumatory and instrumental responses. In the restrained and unrestrained monkey, ESB was supplied by a radio stimulator (Delgado, 1963) with the following stimulus characteristics: constant current, monopolar, cathodal rectangular pulses of 0.5 -msec pulse duration and $100 \mathrm{~Hz}$.

RESULTS AND DISCUSSION

Table 1 shows that in three of the four monkeys, stimulation of 13 points specifically inhibited threatening or grimacing without producing any motor effect, impairing locomotor ability, or disrupting monkey's consumatory, instrumental, or grooming responses. During inhibiting ESB(IESB), the unrestrained monkey showed normal coordinated movement about cage and ran to back of cage when $E$ approached. Fine motor coordination was unaffected by IESB since Monkey A18, unrestrained, groomed another monkey, was groomed, and self-groomed during IESB. In the submissive monkey, ESB inhibited grimacing and vocalizing but did not disrupt head turning to the side that normally occurred when doll contacted its chair. To determine if threats to other stimuli were inhibited, A18 was also tested with the doll, which it threatened 
Table 1

Percentage of Emotional Responses During Inhibiting Electrical Brain Stimulation (IESB) and Effects of IESB on Other Responses

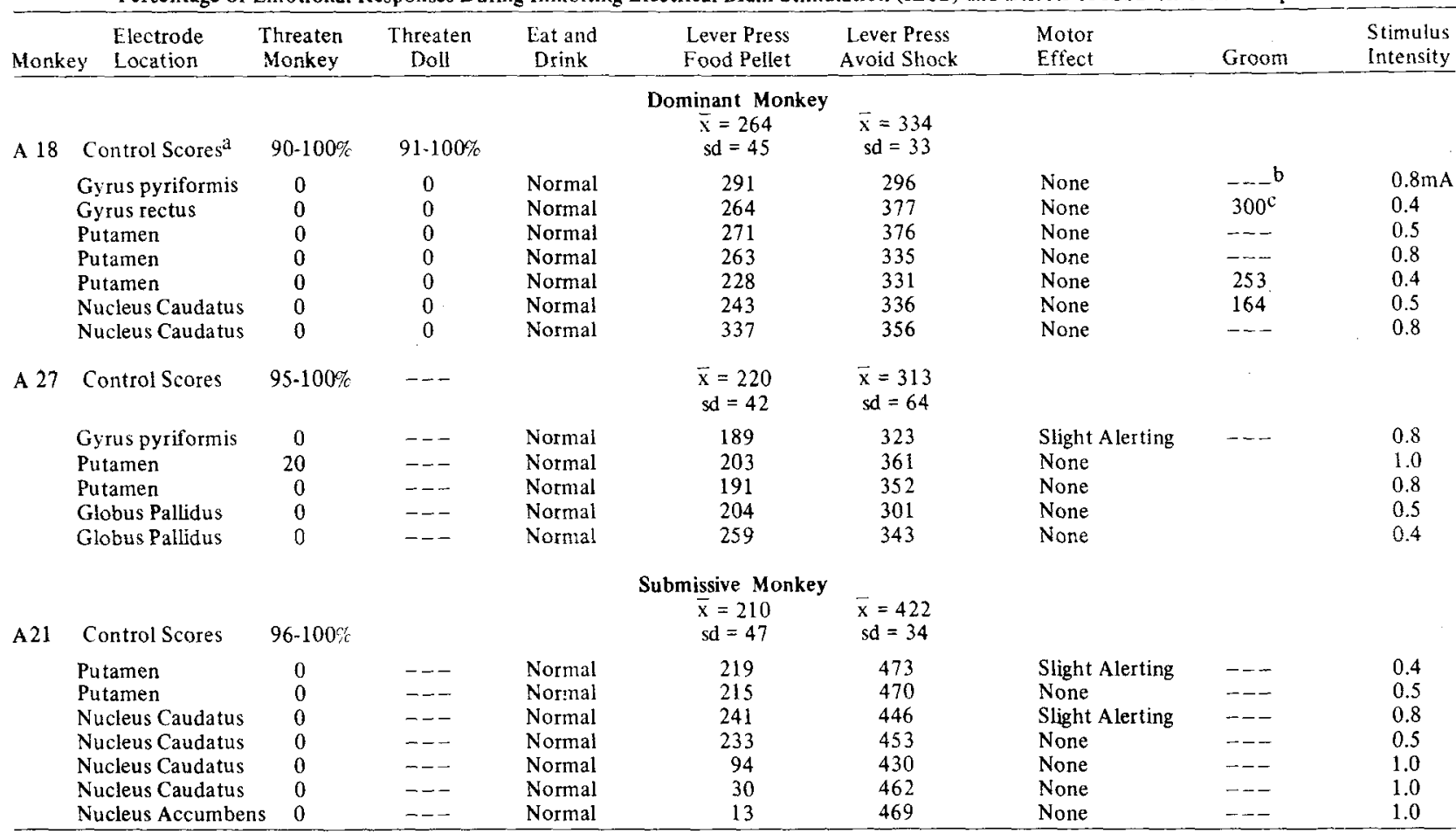

a Range for percentage of emotional responses from daily 60 trial control sessions (shock only). Mean ( $\vec{x}$ ) and standard deviation (sd) computed from 10 five-min records.

S Not tested

$c$ Seconds during which monkey groomed another monkey or self-groomed and IESB occurred 5 sec on/5 sec off.

following shock. ESB inhibited threatening following shock. ESB inhibited threatening to both submissive monkey and doll.

The monkey's facial musculature was not paralyzed by IESB since during IESB each monkey, restrained or unrestrained, drank water and could open its mouth wide enough to bite and place half of a banana in and then chew it. Similarly, the unrestrained monkey drank and placed food pellets in its mouth and chewed pellets during IESB. Therefore, the inhibition of threatening and grimacing did not appear to be a secondary effect, that is, IESB, by paralyzing facial musculature, prevented occurrence of emotional responses.

During shock sessions, monkey's responding can be considered to be motivated by fear or general excitement. Since IESB did not impair the instrumental shock-avoidance performance, we can conclude that IESB did not inhibit emotional responses by raising the threshold for fear or general excitement.

Finally, since IESB did not disrupt two learned tasks-lever pressing for pellets and to avoid shock-we have evidence against the interpretation that IESB inhibited emotional responses by producing general disorientation or disorganization.
Inhibition of emotional and other responses has been produced by electrical stimulation of the amygdala (Fonberg \& Delgado, 1961; Egger \& Flynn, 1963; Fonberg, 1963; Rubinstein \& Delgado, 1963; Delgado, 1964), hippocampus (Siegel \& Flynn, 1968), and caudate nucleus (Rubinstein \& Dalgado, 1963; Dalgado, 1964). The present study confirms the inhibiting effects of caudate stimulation and is the first to report inhibition from any brain area of specific emotional responses without disruption of instrumental, locomotor, consumatory, or grooming behaviors. Although the mechanism of IESB is unknown, evidence is provided that negates interpretations of peripheral muscular impairment, general disorientation, or increase in fear threshold.

\section{REFERENCES}

DELGADO, J. M. R. Evaluation of permanent implantation of electrodes within the brain. Electroencephalography \& Clinical Neurophysiology, 1955, 7,637-644.

DELGADO, J. M. R. Telemetry and telestimulation of the brain. In L. Slater (Ed.), Bio-Telemetry. New York: Pergamon Press, 1963. Pp. 231-249.

DELGADO, J. M. R. Free behavior and brain stimulation. In C. C. Pfeiffer and I. R.
Smythies (Eds.), International review of neurobiology. Vol. 6. New York: Academic Press, 1964. Pp. 349-449.

EGGER, M. D., \& FLYNN, J. P. Effects of electrical stimulation of the amygdala on hypothalamically elicited attack behavior in cats. Journal of Neurophysiology, 1963, 26, 705-720.

FONBERG, E., \& DELGADO, J. M. R. Avoidance and alimentary reactions during amygdala stimulation. Journal of Neurophysiology, 1961, 24, 651-664.

FONBERG, E. The inhibitory role of amygdala stimulation. Acta Biologiae Experimentalis, 1963, 23, 171-180.

PLOTNIK, R., MIR, D., \& DELGADO, J. M. R. Aggression, noxiousness and brain stimulation. In J. P. Scott and B. E. Eleftheriou (Eds.), Physiology of fighting and defeat. Chicago: University of Chicago Press, in press.

RUBINSTEIN, E. H., \& DELGADO, J. M. R. Inhibition induced by forebrain stimulation in the monkey. American Journal of Physiology, $1963,205,941-948$.

SIEGEL, A., \& FLYNN, J. P. Differential effects of electrical stimulation and lesions of the hippocampus and adjacent regions upon attack behavior in cats. Brain Research, 1968, 7 252-267.

\section{NOTE}

1. This research was supported by grants from the Air Force Systems Command, United States Air Force (F 29600-67-C-0058), the Office of Naval Research [609(48)], and the United States Public Health Service (MH-02004). 